\title{
Anknüpfung von Modularisierungsmethoden an ein Wirkmodell modularer Produktstrukturen
}

\author{
Lea-Nadine Schwede ${ }^{1}$, Florian Seiler ${ }^{1}$, Dieter Krause ${ }^{1}$
}

${ }^{1}$ Institute of Product Development and Mechanical Engineering Design (PKT), Hamburg University of Technology (TUHH), Germany

\section{Abstract}

Economic goals, such as costs, are some of the main reasons why companies make changes to their products. To maintain external diversity while reducing internal diversity, modularization is an obvious approach. Therefore, the literature offers a wide range of methods. This paper presents an approach to examine common modularization methods in terms of their impact on economic goals and to correlate this information. In a first step, the various data required to solve this task are linked to each other in a consistent data model. The aim is to create a model-based knowledge base that also indicates the need for action in the development of new methods.

Keywords: Modularization Methods, Impact Model, Economic Effects, ModelBased Systems Engineering

\section{Einleitung und Motivation}

Mit dem Wachstum von Unternehmen wächst oft auch die Anzahl der angebotenen Produktvarianten und somit die externe Vielfalt, da immer mehr Kundenwünsche befriedigt werden sollen. Eine wachsende Anzahl von Produktvarianten hat diverse Auswirkungen auf unterschiedliche Lebensphasen. Beispiele dafür sind ein größerer Lageraufwand der einzelnen Komponenten 
bzw. Module in der Beschaffung (z.B. [1]), die Notwendigkeit mehrerer unterschiedlicher, spezialisierter Werkzeuge in der Produktion (z.B. [2]) oder auch ein erhöhter Schulungsauswand für Mitarbeiter im Vertrieb [3]. Diese unterschiedlichen Auswirkungen in den Lebensphasen haben wiederum negative Auswirkungen auf wirtschaftliche Zielgrößen wie Zeit, Kosten, Qualität und Flexibilität. Die negativen Auswirkungen bewegen Unternehmen unter anderem dazu, Änderungen an ihren Produkten vorzunehmen. Dabei ist die Modularisierung eine sehr gute Möglichkeit, um die interne Vielfalt bei gleichbleibender externer Vielfalt zu reduzieren. Die Auswirkungen von modularisierten Produktstrukturen wurde bereits literaturseitig in einem Wirkmodell modularer Produktstrukturen zusammengestellt $[4,5]$, allerdings ohne das Einbeziehen der Information darüber, welche Modularisierungsmethode jeweils durchgeführt wurde.

\section{Auswahl von Modularisierungsmethoden}

Für die Entwicklung von Produktstrukturen liefert die Literatur eine Vielzahl unterschiedlicher Methoden, von denen einige auch die Modularisierung von Produktstrukturen im Fokus haben. Die Modularisierungsmethoden sind grundlegend ähnlich aufgebaut: Auf die Dekomposition folgt nach der Analyse und Dokumentation der Komponenten und ihrer Kopplungen die Synthese und Reintegration der Komponenten zu Modulen. [6]

Zudem können Modularisierungsmethoden unter anderem in technischfunktional und produktstrategisch unterteilt werden [7]. Zu diesen Ansätzen gehören unter anderem die Theory of Modular Design nach Stone [8], die Design Structure Matrix nach Pimmler und Eppinger [9], die Methode Modular Function Deployment nach Erixon [10] und die Lebensphasenmodularisierung nach Blees [11]. Stone stellt einen Ansatz für die technisch-funktionale Modularisierung bereit. In diesem Ansatz wurden drei unterschiedliche Heuristiken definiert: Umwandlung und Übertragung, dominanter Fluss und Verzweigung. Mithilfe der Heuristiken lassen sich unterschiedliche Konzepte einer modularen Produktstruktur entwickeln, welche anschließend unter dem Einbeziehen von Kundenanforderungen kombiniert werden. Pimmler und Eppinger haben die Design Structure Matrix, welche initial von Steward entwickelt wurde [12], so verwendet, dass diese die Kopplungen zwischen den verschiedenen Komponenten anzeigt. Der Grundgedanke der Verwendung der Matrix ist es, die Komponenten, welche viele Kopplungen zueinander besitzen, in einem Modul zu gruppieren. Dazu werden die matrixbasiert aufgenommenen Kopplungen entlang der Diagonalen in der symmetrischen Matrix mithilfe eines Algorithmus so sortiert, dass sich Gruppierungen ergeben, welche dann Modulvorschläge darstellen. 
Erixon hat die Methode Modular Function Deployment [10] entwickelt. Dabei handelt es sich um einen Produktstrategischen Ansatz. Mithilfe von Modultreibern werden Modulkandidaten definiert. Die Lebensphasenmodularisierung [11] ist Teil des integrierten PKT-Ansatzes zur Entwicklung modularer Produktstrukturen und kombiniert technisch-funktionale mit produktstrategischen Inhalten. Sie baut auf den Ergebnissen der variantengerechten Produktgestaltung auf, welche ebenfalls Teil des integrierten PKT-Ansatzes ist.[6] Unter anderem hat Ripperda [7] ausgewählte Methoden hinsichtlich unterschiedlicher Kriterien in seiner Arbeit untersucht, um die Auswahl der Methoden nach diesen Kriterien zu ermöglichen.

\section{Auswirkungen modularer Produktstrukturen}

Die (Um)Gestaltung von Produktstrukturen bringt unterschiedliche Auswirkungen mit sich. So hat beispielsweise die Verringerung der Komponentenvarianz durch die kommunale Verwendung von Modulen in Produktfamilien in der Produktion geringere Inventarkosten, geringere Umrüstkosten und eine Verringerung der Werkzeugkosten zur Folge [13]. In der Beschaffung führt die kommunale Verwendung von Modulen zur Reduzierung der Anzahl unterschiedlicher Artikelnummern zu der Verkleinerung der Sicherheitsbestände im Lager und somit zur Senkung der Lagerhaltungskosten. Auch die Beschaffungskosten weisen zudem einen mathematischen Zusammenhang zu den Lagerhaltungskosten auf [14]. Viele der in der Literatur, meistens generisch beschriebenen, Auswirkungen, welche eine modularisierte Produktstruktur in den Lebensphasen und auf die wirtschaftlichen Zielgrößen hat, wurden in einem Wirkmodell modularer Produktstrukturen zusammengetragen. Die Modularität von Produktstrukturen wird darin durch die Modularisierungseigenschaften und -merkmale (nach Salvador [15]) definiert, welche durch Kennzahlen wie beispielsweise dem Commonality Index nach Martin und Ishii [16] hinterlegt sind. Die Effekte sind in Effektketten dargestellt und in Primär- und Sekundäreffekte unterteilt. Diese Effektketten führen zu Auswirkungen auf die wirtschaftlichen Zielgrößen. Die Primäreffekte, welche direkt aus der Änderung der Produktstruktur entstehen, sind beispielsweise die Verringerung der Varianz oder die Reduzierung der Anzahl unterschiedlicher Artikelnummern. Die Sekundäreffekte entstehen aus den Primäreffekten und haben dadurch nur noch einen indirekten Zusammenhang zu der Produktstruktur. Beispiele für Sekundäreffekte sind kleinere Inventarkosten, kleinere Sicherheitsbestände und Verringerung der Lagerhaltungskosten. [4,5] 


\subsection{Datenmanagement mit der Unterstützung des Model-Based Sys- tems Engineering}

Die Verwaltung unterschiedlicher Datentypen und deren Schnittstellen wird im Rahmen dieser Arbeit durch das Model-Based Systems Engineering (MBSE) unterstützt, da im Gegensatz zu rein itemzentrierten PLM-Systemen auch übergeordnete Verknüpfungen verarbeitet werden können (u.a. $[17,18])$. Die Stärke des MBSE liegen dabei vor allem darin, dass neben den unterschiedlichen Datenzusammenhänge auch unterschiedliche Datentypen innerhalb eines Objekts berücksichtigt und somit mathematisch betrachtet werden können [19]. Die Verknüpfung der unterschiedlichen Datentypen wird dabei durch die Möglichkeit zur Schnittstellenanbindung in MBSE unterstützt [20]. So können beispielsweise die itemzentrierten Daten aus PLM als zu integrierende Basisdaten einer MBSE-Datenstruktur dienen. Weitere mögliche Schnittstellen sind unter anderem zu dem Austauschformat .csv oder auch zu Matlab vorhanden [21].

Für die Modellierung wird in dieser Arbeit dafür der Cameo Systems Modeler und die Systems Modeling Language (SySML) verwendet. Die Modellierung von Datenzusammenhängen kann dabei in neun unterschiedlichen Diagrammen erfolgen [22], wobei die einzelnen Elemente jeweils nur einmal in einem Modell existieren. Dadurch wird eine Rückverfolgbarkeit gewährleistet und das Änderungsmanagement vereinfacht [23].

\section{Definition der Forschungslücke und des Forschungsziels}

Obwohl die gewünschte Verbesserung der wirtschaftlichen Zielgrößen (Zeit, Kosten, Qualität) oft der Anlass sind, eine Modularisierungsmaßnahme durchzuführen, fehlt bisher eine konkrete Zuordnung von den Modularisierungsmethoden zu wirtschaftlichen Auswirkungen. Dabei sind die unterschiedlichen Lebensphasen in Betracht zu ziehen, um eine unternehmensübergreifende, ideale Ansteuerung der Zielgrößen zu erreichen. Das primäre Forschungsziel ist es daher, eine Wissensbasis zu erstellen. Diese soll es ermöglichen, geeignete Modularisierungsmethoden zu selektieren, um gewollte wirtschaftliche Auswirkungen zielgerichtet zu erreichen. Zudem soll diese Wissensbasis verwendet werden, um durch die modularisierte Produktstruktur nicht abgedeckte wirtschaftliche Auswirkungen zu detektieren. Diese Analysen sollen einen Handlungsbedarf zur Neuentwicklung von Modularisierungsmethoden aufzeigen, die dann diese Lücken füllen. 


\section{Vorgehen zur Verknüpfung von Modularisierungsmethoden an das Wirkmodell modularer Produktstrukturen}

Das Forschungsvorgehen unterteilt sich in drei Abschnitte. Zunächst wird ein Datenmodell entwickelt, welches die relevanten Datenzusammenhänge des betrachteten Systems darstellt. Anschließend erfolgt die Vorstellung eines Ansatzes zur Untersuchung von Modularisierungsmethoden hinsichtlich ihrer Auswirkungen, deren Ergebnisse anschließend in Beziehung gesetzt werden. Abschließend erfolgt die Anknüpfung der Ergebnisse des vorangegangenen Schrittes an das Wirkmodell modularer Produktstrukturen, um die übergeordneten Zusammenhänge zu analysieren.

\subsection{Darstellung der relevanten Datenzusammenhänge in einem Da- tenmodell}

Die Verknüpfung von gängigen Modularisierungsmethoden an das Wirkmodell modularer Produktstrukturen erfolgt über die Modularisierungseigenschaften und -merkmale. Um die Verknüpfung übergeordnet rückverfolgbar und konsistent zu gestalten, wurde ein Datenmodell erstellt, welches die Zusammenhänge der einzelnen Elemente veranschaulicht (Bild 1).

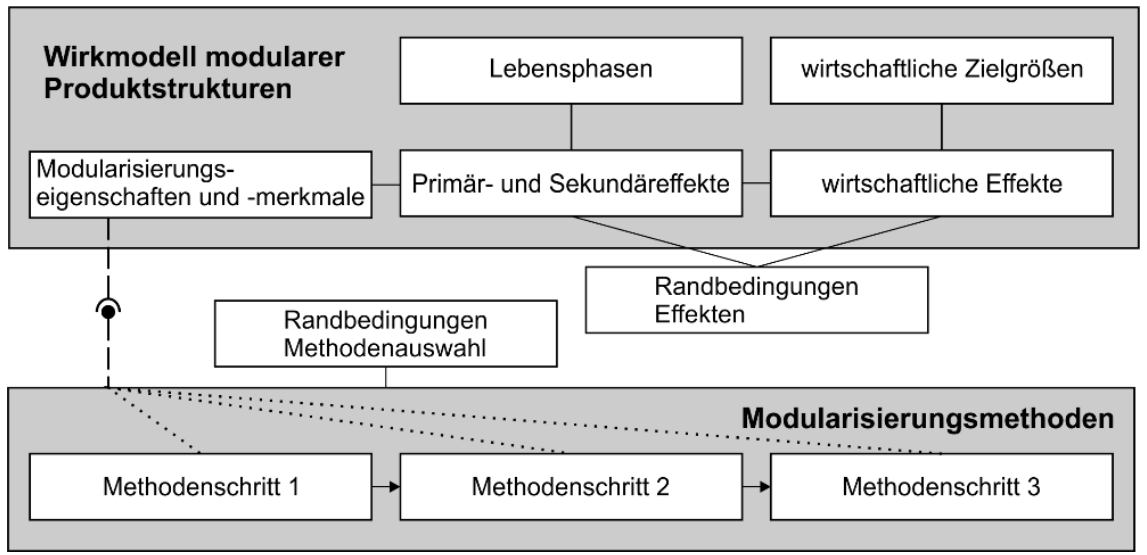

Bild 1: Darstellung des Datenmodells (angelehnt an [24])

Dieses Datenmodell stellt einer Weiterentwicklung des in [24] vorgestellten Datenmodells dar, welches die Zusammenhänge der Elemente in dem Wirkmodell modularer Produktstrukturen mit zusätzlichen Randbedingungen beinhaltet. Das bestehende Datenmodell wird um Modularisierungsmethoden und Randbedingungen zur Methodenauswahl erweitert, wobei die 
hinzugekommene Anknüpfung gestrichelt dargestellt ist. Die Methoden werden in diesem Modell als Prozesse, ähnlich wie Flussdiagramme nach Birkhofer [25] dargestellt, um ggf. einzelne Methodenschritte direkt an die Modularisierungseigenschaften und -merkmale knüpfen zu können (gepunktet dargestellt), falls diese für den Zusammenhang verantwortlich sind. Das durchgängige Datenmodell wurde entwickelt, um zu veranschaulichen, welche dieser beschriebenen Elemente direkt oder auch indirekt miteinander zusammenhängen. Das durchgängige Datenmodell bildet die Grundlage zur Abbildung der Datenzusammenhänge in SysML.

\subsection{Ansatz zur Untersuchung von Modularisierungsmethoden hin- sichtlich ihrer Auswirkungen}

Bevor die Untersuchungen der Modularisierungsmethoden hinsichtlich ihrer Auswirkungen durchgeführt werden können, müssen zunächst die Modularisierungsmaßnahmen an sich untersucht werden. Die Methoden werden miteinander verglichen, um Gemeinsamkeiten und Unterschiede festzustellen. Zudem werden bereits durchgeführte Methodenanalysen herangezogen. Ein wichtiger Schritt ist die Unterteilung der Methoden in ihre Methodenschritte. Anschließend müssen die Methodenschritte hinsichtlich des im jeweiligen Schritt generierten Outputs und den daraus folgenden Auswirkungen untersucht werden. Die Sicht auf die Methodenschritte der einzelnen Methoden muss in beiden Untersuchungen, welche im Folgenden vorgestellt werden, berücksichtigt werden. Um die Auswirkungen von Modularisierungsmethoden zu analysieren, werden zwei unterschiedliche Untersuchungen durchgeführt (Bild 2) und deren Ergebnisse anschließend miteinander in Beziehung gesetzt.
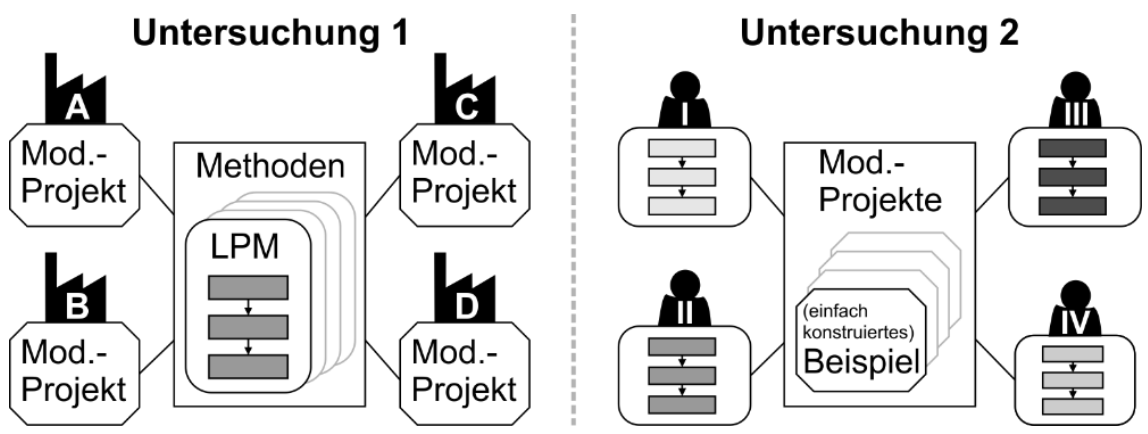

Bild 2: Darstellung der zwei unterschiedlichen Untersuchungen 
Für die erste Untersuchung (Bild 2, links) werden Modularisierungsprojekte untersucht, in welchen dieselbe Modularisierungsmethode bei unterschiedlichen Unternehmen $(A, B, C, D)$ durchgeführt wurde, die jeweils unterschiedliche Unternehmensrandbedingungen aufweisen. Da die Lebensphasenmodularisierung (LPM) nach Blees in unterschiedlichen Projekten durchgeführt wurde, kann an dieser Stelle von einer hinreichenden, von den Autoren einsehbaren Datengrundlage ausgegangen werden. Randbedingungen können in diesem Fall unterschiedliche Fertigungsarten (z.B. Einzel/Serienfertigung), Branchen (z.B. Anlagen-/und Maschinenbau) und auch das Produkt bzw. die Produktfamilie, welche das jeweilige Unternehmen herstellt, sein. Die Auswirkungen der Durchführung der Lebensphasenmodularisierung kann über Kennzahlen der Modularisierungseigenschaften und -merkmale berechnet werden. Die Kennzahlen der Produktfamilie vor der Modularisierung und die Kennzahlen der Produktfamilie nach der Modularisierung können miteinander verglichen werden, um die Veränderungen der Modularisierungseigenschaften und -merkmale feststellen zu können. Das Ziel dieser Untersuchung ist es, herauszufinden wozu ein und dieselbe Modularisierungsmethode bei unterschiedlichen Modularisierungsprojekten geführt hat. Durch die Untersuchung mehrerer Modularisierungsprojekte lassen sich Tendenzen für die Auswirkungen und deren Zusammenhang zu Randbedingungen erkennen. Es soll im Rahmen der Auswertung dieser Untersuchung zudem ein Vorgehen entwickelt werden, wie weitere Modularisierungsprojekte untersucht werden können. Langfristig soll die initial untersuchte Modularisierungsmethode durch ein Modularisierungscluster ersetzt werden. Dasselbe Vorgehen kann auch mit anderen Modularisierungsmethoden durchgeführt werden. Die weiteren Untersuchungen hängen dabei stark von der Dokumentation der Durchführung der Modularisierungsmethoden ab.

Es muss davon dennoch ausgegangen werden, dass ein und dasselbe Modularisierungsprojekt nicht mit unterschiedlichen Modularisierungsmethoden unter denselben Randbedingungen in der Industrie durchgeführt wurde. Wenn allerdings weitere Modularisierungsmethoden untersucht werden sollen, bieten sich aufgrund deren Vergleichbarkeit Modularisierungsprojekte an, welche unter ähnlichen Unternehmensrandbedingungen stattgefunden haben. Für ein Unternehmen, welches vor der Entscheidung steht, welche Modularisierungsmethode am besten zu ihrem Anwendungsfall passt und zudem gewünschte Auswirkungen hat, wie beispielsweise die Reduzierung der Durchlaufzeiten in der Produktion oder die Minimierung der Lagerhaltungskosten in der Beschaffung ist eine Untersuchung der gängigen Modularisierungsmethoden hinsichtlich ihrer Unterschiede bezogen auf die Auswirkungen unter exakt gleichen Randbedingungen sinnvoll. Die Auswirkungen unterschiedlicher Modularisierungsmethoden, bezogen auf dasselbe Anwendungsbeispiel, lässt 
sich in der Industrie nur schwer überprüfen. Aus diesem Grund wird im Rahmen eines Experiments ein einfaches Produktbeispiel mit festgelegten Unternehmensrandbedingungen definiert, auf welches dann von unterschiedlichen Probanden (I, II, III, IV) unterschiedliche Modularisierungsmethoden angewendet werden (Bild 2, rechts). Für die jeweils entstandenen modularen Produktstrukturen werden wiederum die Kennzahlen der Modularisierungseigenschaften und Merkmale berechnet. Das langfristige Ziel in diesem Fall ist der Austausch des Anwendungsbeispiels bzw. die Änderung der Unternehmensrandbedingungen und die erneute Durchführung der Experimente mit neuen Probanden.

\subsection{Analyse der Verknüpfung von Modularisierungsmethoden und dem Wirkmodell modularer Produktstrukturen}

Nachdem die Zusammenhänge zwischen den Modularisierungsmethoden (bzw. deren Methodenschritte) und ihren Auswirkungen auf die Modularisierungseigenschaften und -merkmale modularer Produktstrukturen untersucht wurden, kann die Anknüpfung der Modularisierungsmethoden an das Wirkmodell modularer Produktstrukturen, wie in dem Datenmodell (Bild 1) beschrieben, durchgeführt werden (Bild 3).

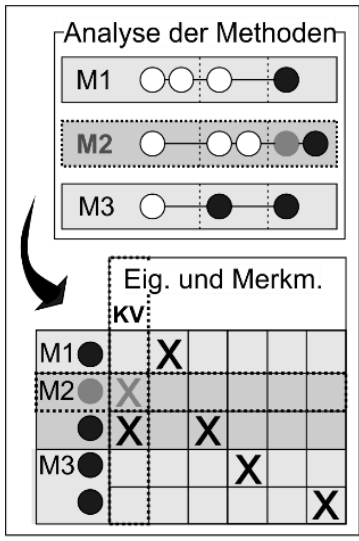

M Modularisierungsmethoden KV Kommunale Verwendung
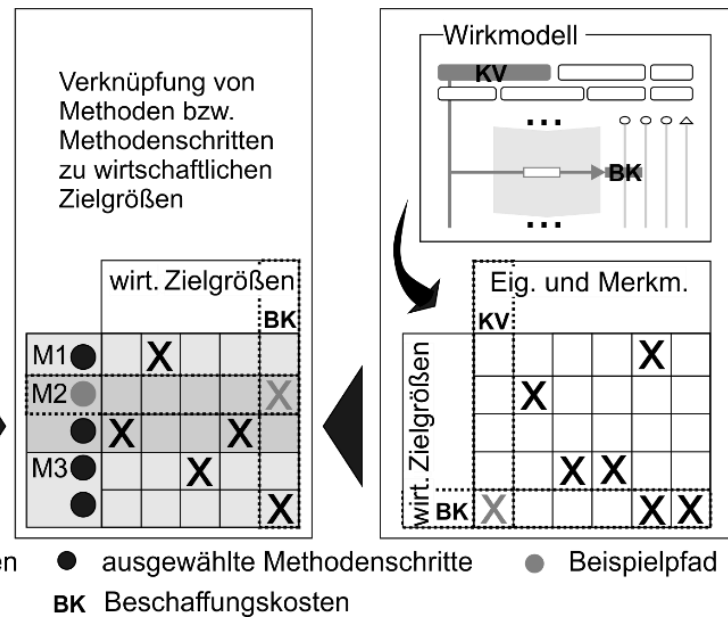

Bild 3: Vorgehen zur Verknüpfung von Methoden und Wirkmodell

Die Modularisierungseigenschaften und -merkmale stellen, wie bereits in Bild 1 erkennbar, den Anknüpfungspunkt zum Wirkmodell modularer Produktstrukturen dar. Somit werden die Methoden bzw. einzelne Methodenschritte über die Modularisierungseigenschaften und -merkmale, sowie den Effekten in den Lebensphasen an die wirtschaftlichen Zielgrößen übergeordnet 
modellbasiert verknüpft. In Bild 3 ist ein Beispielspfad in dunkelgrau, gestrichelt dargestellt: Während der Analyse der Modularisierungsmethoden (Bild 3, links) wurde der vierte Methodenschritt der Methode 2 (M2) als derjenige identifiziert, welcher einen Einfluss auf die kommunale Verwendung (KV) von Modulen hat. Aus dem Wirkmodell modularer Produktstrukturen ist bekannt, welche Auswirkungen auf die wirtschaftlichen Zielgrößen durch die Kommunale Verwendung entstehen (im Beispiel: Beschaffungskosten (BK), Bild 3, rechts). Die Informationen aus der Analyse der Methoden und aus dem Wirkmodell modularer Produktstrukturen werden anschließend so zusammengebracht, dass eine Verknüpfung zwischen ihnen hergestellt werden kann (Bild 3, Mitte). Mithilfe der Modellierung in SysML lassen sich die übergeordneten Zusammenhänge zurückverfolgen. Durch die modellbasierte Umsetzung lassen sich die mehrstufigen Zusammenhänge analysieren. Die Software Cameo Systems Modeler stellt zu diesem Zweck unterschiedliche Analyse- und Berechnungstools bereit. Hier kann beispielsweise ausgegeben werden, wie oft eine Eigenschaft bzw. ein Merkmal des Wirkmodells modularer Produktstrukturen durch Modularisierungsmethoden angesteuert wurde. Auch wirtschaftliche Zielgrößen, welche eventuell durch die Anwendung einer bestimmten Methode nicht angesteuert werden, lassen sich im Rahmen einer softwareinternen Simulation anzeigen. Diese nichtangesteuerten Auswirkungen decken neue Forschungsfelder in der Methodenentwicklung auf. Auch die Methoden und deren Überschneidungen, beispielsweise durch die Verwendung der gleichen Werkzeuge in einzelnen Methodenschritten, werden in dem Modell dargestellt. Dadurch kann herausgefunden werden, welche Methodenanteile eventuell miteinander kombiniert werden können, um mehr Auswirkungen zu erzielen. In einem abschließenden Schritt wird das gesammelte Wissen über die Datenzusammenhänge von Methoden und deren Auswirkungen so gespeichert, dass es für die zukünftige Methodenentwicklung genutzt werden kann. Deshalb wird eine modellbasierte Übersicht über Methoden und deren Auswirkungen, aufbauend auf einem durchgängigen Datenmodell unter Einbezug von möglichen Anwendern entwickelt, um die Benutzerfreundlichkeit und den Nutzen bewerten zu können.

\section{Diskussion und Ausblick}

Das Modellieren der Datenzusammenhänge aus dem Wirkmodell modularer Produktstrukturen und der Modularisierungsmethoden kann mithilfe des MBSE in SysML durchgeführt werden. Unterschiedliche Daten, wie bspw. Produktstrukturen, Prozesse und Methodenschritte können dabei miteinander verknüpft werden. Somit können bestehende produktbezogene Daten aus PLM-Systemen in das Datenmodell integriert werden. Die Durchgängigkeit ist durch eine konsistente Datenstruktur gewährleistet, welche erweiterbar und aktualisierbar ist, falls beispielsweise eine weitere Methode aufgenommen 
werden soll oder im Rahmen der Forschung neue Effekte zum Wirkmodell modularer Produktstrukturen hinzukommen. Vor Allem der Unterschied zwischen den dokumentierten Auswirkungen in der Literatur, oder auch in den Methoden-beschreibungen und den Informationen der Auswirkungen direkt aus dem Unternehmen ist dabei zu beachten. Die Datenzusammenhänge können sowohl als Wissensbasis, oder auch zu Rekombination von Methoden verwendet werden. Zudem können sie auch als Basis für methodenunabhängige Entwicklungen dienen, wie z.B. einem dynamischen Produktkonfigurator, mit Hilfe dessen das Wissen aus der vorangegangenen Modularisierung den Kunden intuitiv zur Verfügung gestellt werden kann. Die daraus resultierenden Konfigurationsdaten können dazu genutzt werden, die Effizienz der modularen Architektur zu evaluieren und auf dieser Basis weiterzuentwickeln. Ebenfalls können dadurch weitere Defizite der Modularisierungsmethoden aufgedeckt werden, die wiederum wie oben beschrieben zur Weiterentwicklung der Modularisierungsmethoden dienen können.

\section{Danksagung}

Die dargestellten Ergebnisse wurden im Rahmen des Forschungsprojektes "WiMo 2 - Entwicklung eines Wirkmodells der Eigenschaften modularer Produktstrukturen zur Bewertung methodischer Ansätze" [GZ: KR 3478/7-2] entwickelt, das von der Deutschen Forschungsgemeinschaft (DFG) gefördert wird.

\section{Literatur}

[1] Dogramaci, A., "Design of Common Components Considering Implications of Inventory Costs and Forecasting" A I I E Transactions, 2007, 129-135.

[2] Perera, H. S. C., Nagarur, N., und Tabucanon, M. T., "Component part standardization. A way to reduce the life-cycle costs of products", International journal of production economics, 60, 1999, 109-116.

[3] Harland, P. E. und Uddin, Z., "Effects of product platform development. Fostering lean product development and production", International journal of product development: IJPD 19, 5/6, 2014, 259-285.

[4] Hackl, J. und Krause, D., "Effects of Modular Product Structures on Life Phases and Economic Factors", Proceedings of the 14th International Design Conference - Design 2016, Dubrovnik, Croatia, 2016 12851294. 
[5] Hackl, J. und Krause, D., "Towards an Impact Model of modular Product Structures", Proceedings of the 21st International Conference on Engineering Design - ICED17, Vancouver, Canada, 2017, 151-160.

[6] Krause, D. und Gebhardt, N., "Methodische Entwicklung modularer Produktfamilien. Hohe Produktvielfalt beherrschbar entwickeln." Springer Vieweg, Berlin, 2018.

[7] Krause, D. und Ripperda, S., "An Assessment of methodical Approaches to support the Development of Modular Product Families", International Conference on Engineering Design - ICED13, Seoul, Korea, 2013.

[8] Stone, R. B., "Towards a Theory of modular Design", Dissertation, The University of Texas at Austin, 1997.

[9] Pimmler, T. U. und Eppinger, S. D., "Integration Analysis of Product Decompositions", ASME Design Theory and Methodology Conference, Minneapolis, MN, 1994.

[10] Erixon, G. "Modular Function Deployment - A Method for Product Modularisation", Dissertation, The Royal Insitute of Technology, Sweden, 1998.

[11] Blees, C., „Eine Methode zur Entwicklung modularer Produktfamilien.", Dissertation, Technische Universität Hamburg-Harburg, 2011.

[12] Steward, D., "The Design Structure System: A Method for Managing the Design of Complex Systems", IEEE Transactions on Engineering Management, Vol. EM-28, No. 3. 1981, 71-74.

[13] Ulrich, K., "The role of product architecture in the manufacturing firm", Research Policy 24, 3, 1995, 419-440.

[14] Hutzschenreuter, T., „Allgemeine Betriebswirtschaftslehre. Grundlagen mit zahlreichen Praxisbeispielen. Lehrbuch", Springer Gabler, Wiesbaden, 2015.

[15] Salvador, F., "Toward a Product System Modularity Construct: Literature Review and Reconceptualization", IEEE Transactions on Engineering Management 54, 2, 2007, 219-240. 
[16] Martin, M. V. und Ishii, K., "Design for Variety: Development of Complexity Indices and Design Charts", ASME Design Engineering Technical Conferences, Sacramento, CA, 1997.

[17] Mueggo, C. und Pfenning, M., „Die Rolle von MBSE und PLM im Industrial Internet", Tag des Systems Engineering 2015, 2015.

[18] Eigner, M., Gilz, T., und Zafirov, R., "Proposal for functional product description as part of a PLM solution in interdisciplinary product development", Proceedings of the 12th International Design Conference - Design 2012, Dubrovnik, Croatia, 2012, 1667-1676.

[19] Liebisch, M. „Aspektorientierte Datenhaltung in Produktkonfiguratoren Anforderungen, Konzepte und Realisierung", Dissertation, FriedrichSchiller-Universität Jena, 2014.

[20] Sendler, U. und Weilkiens, T., "Modellbasierte Systementwicklung: Was Sie schon immer über MBSE, PLM und Industrie 4.0 wissen sollten (I)", Tag des Systems Engineering 2013, 2013, 303-314.

[21] No Magic, "Cameo Systems Modeler Documentation 18.3", https://docs.nomagic.com/display/CSM183, abgerufen am 10.08.2019.

[22] Holt, J., Perry, S. A., und Brownsword, M., "Model-based requirements engineering", IET professional applications of computing series 9, Institution of Engineering and Technology, London, 2012.

[23] Bursac, N., "Model Based Systems Engineering zur Unterstützung der Baukastenentwicklung im Kontext der Frühen Phase der Produktgenerationsentwicklung", Dissertation, Karlsruher Institut für Technologie, 2016.

[24] Schwede, L.-N. et al., "Consistent Modelling of the Impact Model of Modular Product Structures with Linking Boundary Conditions in SysML", Proceedings of the 22nd International Conference on Engineering Design - ICED19, Delft, Niederlande, 2019.

[25] Birkhofer, H. et al., "Cleaning up Design Methods - Describing Methods Completely and Standardised", International Design Conference - Design 2002, Dubrovnik, Crotaia, 2002, 17-22. 